UVX 2012, 01009 (2013)

DOI: $10.1051 /$ uvx/201301009

(C) Owned by the authors, published by EDP Sciences, 2013

\title{
LUNEX5: Toward an advanced FEL project
}

\author{
M.E. Couprie ${ }^{1}$, C. Benabderrahmane ${ }^{1}$, P. Betinelli ${ }^{1}$, F. Bouvet ${ }^{1}$, A. Buteau $^{1}$, \\ L. Cassinari ${ }^{1}$, J. Daillant ${ }^{1}$, J.C. Denard ${ }^{1}$, P. Eymard ${ }^{1}$, B. Gagey ${ }^{1}$, \\ C. Herbeaux ${ }^{1}$, M. Labat ${ }^{1}$, A. Lestrade ${ }^{1}$, A. Loulergue ${ }^{1}$, P. Marchand ${ }^{1}$, \\ J.L. Marlats ${ }^{1}$, C. Miron ${ }^{1}$, P. Morin ${ }^{1}$, A. Nadji ${ }^{1}$, F. Polack ${ }^{1}$, J.B. Pruvost ${ }^{1}$, \\ F. Ribeiro1, J.P. Ricaud', P. Roy¹, T. Tanikawa1 , R. Roux², S. Bielawski', \\ C. Evain ${ }^{3}$, C. Szwaj ${ }^{3}$, G. Lambert ${ }^{4}$, A. Lifschitz ${ }^{4}$, V. Malka ${ }^{4}$, R. Lehe ${ }^{4}$,

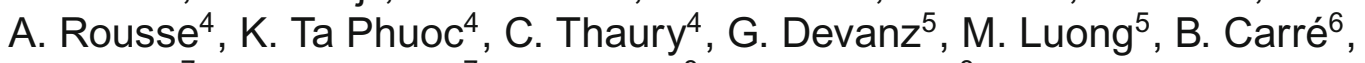 \\ G. LeBec ${ }^{7}$, L. Farvacque ${ }^{7}$, A. Dubois ${ }^{8}$ and J. Lüning ${ }^{8}$ \\ ${ }^{1}$ Synchrotron SOLEIL, Saint-Aubin, France \\ ${ }^{2}$ Laboratoire de l'Accélérateur Linéaire, Orsay, France \\ ${ }^{3}$ PhLAMI CERLA, Lille, France \\ ${ }^{4}$ Laboratoire d'Optique Appliquée, Palaiseau, France \\ ${ }^{5}$ CEAIDSMIIRFU/SACM, Saclay, France \\ ${ }^{6}$ CEAIDSMIIRAMISISPAM, Saclay, France \\ ${ }^{7}$ ESRF, Grenoble, France \\ ${ }^{8}$ LCPMR, Paris-VI, France
}

\begin{abstract}
LUNEX5 (free electron Laser Using a New accelerator for the Exploitation of X-ray radiation of 5th generation) aims at investigating the production of short, intense, and coherent pulses in the soft x-ray region. The single Free Electron Laser (FEL) line fed either by a superconducting Linear Accelerator of $400 \mathrm{MeV}$ or a $0.4-1 \mathrm{GeV}$ Laser Wake Field Accelerator (LWFA) enables seeding (High order Harmonic in Gas seeding and Echo Enabled Harmonic Generation) for the advanced fourth generation source with the conventional accelerator, and single spike operation for the fifth generation source with the LWFA. Two pilot user experiments for time-resolved studies of isolated species and solid state matter will take benefit of LUNEX5 FEL.
\end{abstract}

\section{INTRODUCTION}

Accelerator based X-ray light sources rely on the emission of synchrotron radiation by relativistic electrons (of normalised energy g) in bending magnets and in periodic permanent magnetic structures, such as undulators. The so-called first generation took advantage of the parasitic synchrotron radiation emitted in the storage rings initially built for high energy physics. The second generation was developed on dedicated storage ring accelerators. The third generation arose with reduced emittances (product of the beam size by the divergence) and high number of installed undulators leading to a brightness increase and a partial transverse coherence. Indeed, a planar undulator creating a sinusoidal vertical field (amplitude Bo, period $\lambda \mathrm{o}$ ), emits on axis at the resonant wavelength 1 and its odd harmonics on axis: $\lambda=\lambda \mathrm{o}(1+\mathrm{K} 2 / 2) / 2 \mathrm{~g} 2$ with $\mathrm{K}=0.94 \lambda \mathrm{o}(\mathrm{cm}) \mathrm{Bo}(\mathrm{T})$, as a result from the interferences between the different periods. Fourth Generation Light Sources ( $4 \mathrm{G})$ enable longitudinal coherence, by setting in phase the emitting electrons thanks to the FEL process. A light wave of wavelength $\lambda$ (spontaneous

This is an Open Access article distributed under the terms of the Creative Commons Attribution License 2.0, which permits unrestricted use, distribution, and reproduction in any medium, provided the original work is properly cited. 


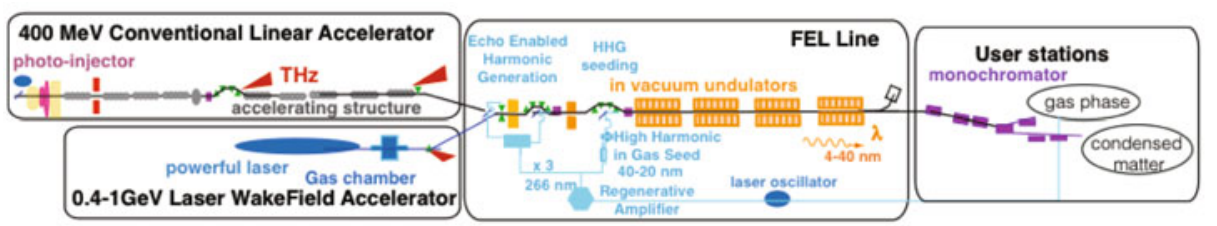

Figure 1. LUNEX5 scheme. From the left : electron gun, superconducting accelerating sections (grey) with the compression chicane in between, LWFA chamber, two echo chicanes and modulator-, radiator-undulators, monochromator, pilot user experimental stations.

emission progressing along the undulator or stored in an optical cavity, or external seed) interacts with the electron bunch in the undulator, inducing an energy modulation of the electrons; which is gradually transformed into density modulation at the wavelength $\lambda$ and leads to a coherent radiation emission. Since the first FEL (MARK-III, Stanford, USA) in the infra-red in an oscillator configuration 1977 [1], two FEL LCLS [2] (Stanford, USA) and SACLA [3] (Harima, Japan) on linear accelerator are now presently operating in the $1 \AA$ region with $100-10 \mathrm{fs}$ pulse and GW peak power. In the Self Amplified Spontaneous Emission configuration where the spontaneous emission progressing along the undulator is amplified towards saturation [4], the uncorrelated trains of radiation resulting from the interaction of electrons progressing jointly with the previously emitted spontaneous radiation lead to spiky longitudinal and temporal distributions, apart from single spike operation for low charge regime [5]. Seeding either with amplified spontaneous emission from first stage undulators sent through a monochromator (so-called self seeding [6]) or with an external laser or a short wavelength coherent light source, such as High order Harmonics generated in gas, allows the saturation length to be shortened, the jitter to be reduced, and the longitudinal coherence to be improved [7-9]. The Echo Enabled Harmonic Generation (EEHG) scheme with a double electron-laser interaction can push the spectral range towards shorter wavelengths when operating on a high order harmonic of the seed wavelength [10-15]. The so-called Fifth Generation $(5 \mathrm{G})$ replacing the conventional linear by a Laser Wakefield Accelerator (LWFA) providing $\mathrm{GV} / \mathrm{m}$ acceleration with very short bunches $[16,17]$ is under progress with first observation of the spontaneous undulator radiation [18-20] and FEL projects [21].

\section{LUNEX5 CONSTITUTING ELEMENTS}

LUNEX5 (see Figure 1) consists in a $400 \mathrm{MeV}$ conventional superconducting accelerator and a 0.3 $1 \mathrm{GeV}$. LWFA accelerator, both feeding a common FEL line. LUNEX5 aims at investigating the production of short, intense, and coherent pulses in the soft x-ray region on advanced 4GLS with the latest seeding schemes with a Conventional Linear Accelerator (CLA) with ultra-short pulses (of a few fs pulse duration) and on 5GLS first for qualifying LWFA with a FEL application, enabling to provide shorter FEL pulses.

\subsection{The conventional accelerator (CLA)}

The CLA includes a superconducting L band linac with a Desy-Zeuthen type photo-gun with a dedicated laser system, a $1.3 \mathrm{GHz}$ cryomodule for $200 \mathrm{MeV}$ acceleration, a third harmonic cavity, a laser heater, a magnetic chicane a diagnostic section, a second $1.3 \mathrm{GHz}$ accelerating module up to $400 \mathrm{MeV}$ and the space for a possible additional third module (see fig. 2). The superconductive cavities will be modified for future $\mathrm{CW}$ operation, the first nominal case of operation being at $50 \mathrm{~Hz} .1 .3 \mathrm{GHz}$ solid state amplifiers will be developed at SOLEIL. 
UVX 2012

\begin{tabular}{|c|c|c|c|c|c|}
\hline Undulator configuration & $\begin{array}{l}\text { Amplifier } \\
\text { @ } 20 \mathrm{~nm}\end{array}$ & $\begin{array}{l}\text { Cascade } \\
\text { @ } 20 \mathrm{~nm}\end{array}$ & $\begin{array}{c}\text { EEHG @ } \\
20 \text { nm }\end{array}$ & $\begin{array}{c}\text { Cascade @ } \\
12 \text { nm }\end{array}$ & $\begin{array}{c}\text { EEHG @ } \\
12 \mathrm{~nm}\end{array}$ \\
\hline Number of UM closed & 0 & 0 & 2 & 0 & 2 \\
\hline Number of UR closed & 3 @ 20 nm & $\begin{array}{l}1 @ 40 \mathrm{~nm} \\
3 @ 20 \mathrm{~nm}\end{array}$ & 2@20 nm & $\begin{array}{l}1 @ 24 \mathrm{~nm} \\
1 @ 36 \mathrm{~nm}\end{array}$ & 1 @36nm \\
\hline \multicolumn{6}{|l|}{ Seed configuration } \\
\hline Wavelength [nm] & 20 & 40 & 266 & 24 & 266 \\
\hline Peak power [MW] & 0.001 & 0.01 & $15-30$ & 0.150 & $15-88$ \\
\hline \multicolumn{6}{|l|}{ Output radiation properties } \\
\hline Fundamental wavelength [nm] & 20 & 20 & 20 & 12 & 12 \\
\hline Photon energy $[\mathrm{eV}]$ & 62 & 62 & 62 & 100 & 100 \\
\hline Peak power [MW] & 54 & 270 & 70 & 50 & 30 \\
\hline Pulse duration [fs-fwhm] & 30 & 17 & 24 & 35 & 7 \\
\hline Pulse energy $[\mu \mathrm{J}]$ & 1.6 & 5 & 2 & 2 & 0.2 \\
\hline Number of photons per pulse & $1.710^{11}$ & $510^{11}$ & $210^{11}$ & $1.110^{11}$ & $1.310^{10}$ \\
\hline Repetition rate [Hz] & 50 & 50 & 50 & 50 & 50 \\
\hline Average power $[\mu \mathrm{W}]$ & 80 & 250 & 100 & 93 & 10 \\
\hline Relative bandwidth (\%) & 0.04 & 0.1 & 5 & 3 & $8 \mathrm{e}$ \\
\hline Beam size [ $\mu \mathrm{m}-\mathrm{rms}]$ & 120 & 300 & 140 & 200 & 60 \\
\hline Beam divergence [ $\mu \mathrm{rad}]$ & 70 & 200 & 40 & 250 & 50 \\
\hline $\begin{array}{l}\text { Peak brightness } \\
{\left[\mathrm{ph} / \mathrm{s} / \mathrm{mm}^{2} / \mathrm{mrad}^{2} / 0.1 \% \text { b.w.] }\right.}\end{array}$ & $1.910^{29}$ & $7.510^{27}$ & $4.510^{29}$ & $3.610^{27}$ & $2.410^{29}$ \\
\hline Average brightness & $3.110^{17}$ & $6.810^{15}$ & $5.710^{11}$ & $6.810^{15}$ & $9.110^{16}$ \\
\hline Peak power@ @3 [kW] & 300 & 2500 & 500 & 2000 & 30 \\
\hline Peak power@ @5 [kW] & 25 & & 8 & 400 & 6 \\
\hline
\end{tabular}

Figure 2. Performances of the LUNEX5 FELs in various configurations. UM = modulator, UR = radiator. Electron beam energy $=400 \mathrm{MeV}$.

\subsection{The plasma accelerator (LWFA)}

Lasers from CILEX (Centre Interdisciplinaire de Lumière Extrême) with APOLLON 10 PW laser and its "proximity centers" such as the $60 \mathrm{TW}$ laser of LOA... will be used for the LWFA before a dedicated one.

\subsection{The FEL line}

Transport to FEL line is obtained with variable permanent magnet quadrupoles. The FEL line comprises transport magnetic elements, dump dipole and in-vacuum undulators with 30 (modulators) or $15 \mathrm{~mm}$ (radiators), with a cryogenic option 15 . At $3 \mathrm{~mm}$ gap, a peak field of $1.5 \mathrm{~T}(1.7 \mathrm{~T})$ will be produced at room temperature with $\mathrm{NdFeB}$ magnet (at $77 \mathrm{~K}$ with $\mathrm{PrFeB}$ magnets) for a $15 \mathrm{~mm}$ period. The seeding laser system with a regenerative amplifier will be used for producing the High order Harmonics in Gas (HHG) generated in a gas cell, for the Echo scheme (after tripling) and for the pump probe experiments of the pilot users.

\subsection{The pilot experiments}

The FEL light will then hit a first toroidal mirror (to be interchanged with a second one for the echo source point) for focusing on the exit slit, it will then pass in a double rotation monochromator operating in the Petersen mode to select and stabilise the photon energy and through two focusing mirror systems to be dispatched on the two experimental set-ups. Diagnostics will consist in standard bunch charge 


\section{Web of Conferences}
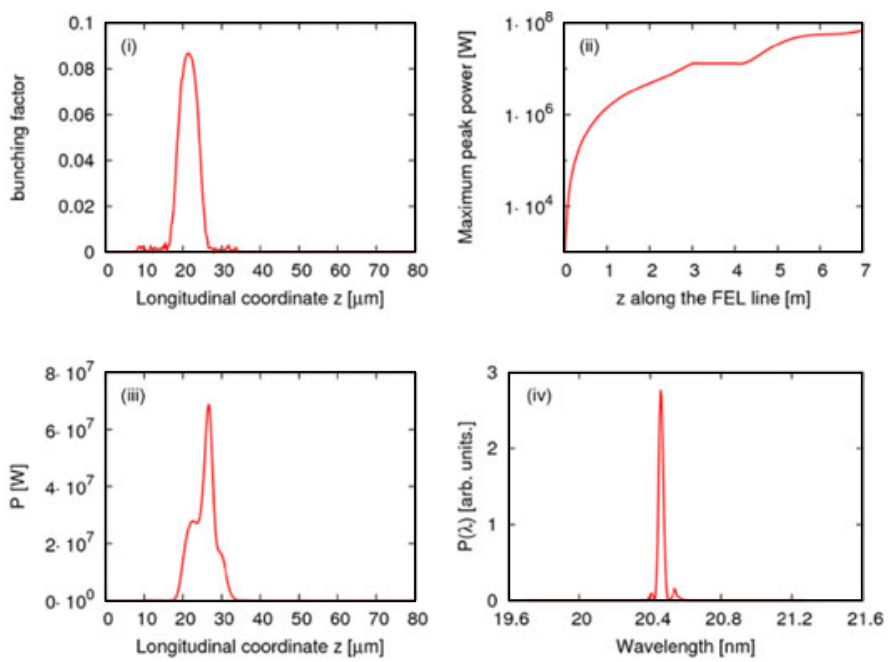

Figure 3. Performances at $20 \mathrm{~nm}$ in the EEHG configuration. (i) Bunching factor at radiator entrance. (ii) Maximum peak power vs undulator radiator sections. Longitudinal (iii) power and (iv) spectral profile after 2 sections of radiators. Seed: $30 \mathrm{fs}-\mathrm{FWHM}$ pulse with $15 \mathrm{MW}$ and $30 \mathrm{MW}$ peak power at $266 \mathrm{~nm}$. Undulator setting: UM1: on, UM2: on, UR1 and UR2: on at $20 \mathrm{~nm}, \mathrm{UR} 3$, UR4 off.

monitors, optical Transition Radiation and fluorescent screens for beam transverse dimensions, standard stripline Beam Position Monitors (BPM) for the linac and cavity BPMs16 for the undulator sections, a beam arrival time monitor, bunch length monitors and an RF deflecting cavity.

\section{LUNEX5 PERFORMANCES}

\subsection{CLA performances}

Figure 2 summarizes the expected performances of LUNEX5 FELs in the various configurations based on CLA. The spectral range covers the $4-40 \mathrm{~nm}$ with the first, third and fifth harmonics, with fundamental wavelength peak power between 10 and $100 \mathrm{MW}$, corresponding to more than $10^{11}$ photons/pulse and $10^{27}$ peak brightness and harmonic wavelength peak power decreasing from $1 \mathrm{MW}$ to a few hundred W. Each wavelength can be obtained with different configurations (amplifier configuration, cascade with a High Order Harmonic seed, echo with $266 \mathrm{~nm}$ lasers...). The FEL saturates earlier in the echo $(7 \mathrm{~m})$ case than in the cascade one $(11 \mathrm{~m})$, with slightly lower power $(65 \mathrm{MW}$ versus $0.27 \mathrm{GW}$ ), longer pulses ( $24 \mathrm{fs}$ versus $17 \mathrm{fs}$ ). Advanced 4GLS FEL tunability is achieved by a combined variation of the seed wavelength and the undulator gaps.

An example of GENESIS calculation of the FEL performances in the EEHG configuration at $20 \mathrm{~nm}$ is presented in Figure 3. The inserts in the Figure reveal the good longitudinal coherence (single spike in the temporal and spectral domain, Fourier limited pulse) and the efficient exponential growth in the two sections undulator.

\subsection{CLA performances}

Figure 4 shows the expected performances of the $5 \mathrm{G}$ FEL in the optimistic case of $0.1 \%$ energy spread.

But the $5 \mathrm{G}$ FEL performances critically depend on the electron beam performances and on the optimization of the transport line. Figure 5 shows for instance the effect on the FEL performances of a bunch length increase, directly inducing a peak current drop: such degradation of the electron beam 
UVX 2012

\begin{tabular}{|c|c|c|c|}
\hline Electron beam & & & \\
\hline Energy (MeV) & 400 & 800 & 1000 \\
\hline Relative energy spread & 0.001 & 0.001 & 0.001 \\
\hline Emittance (п.mm.mrad) & 1 & 1 & 1 \\
\hline Charge $(\mathrm{pC})$ & 50 & 50 & 50 \\
\hline Length (fs-rms) & $2 / 20$ & $2 / 20$ & $2 / 20$ \\
\hline Peak current $(\mathrm{kA})$ & $10 / 1$ & $10 / 1$ & $10 / 1$ \\
\hline \multicolumn{4}{|l|}{ Undulator } \\
\hline Deflection parameter & 1.088 & 2.8 & 0.74 \\
\hline Resonant wavelength $(\mathrm{nm})$ & 19.5 & 15 & 2.5 \\
\hline \multicolumn{4}{|l|}{ Seed } \\
\hline Wavelength (nm) & 19.5 & 15 & - \\
\hline Duration (fs-FWHM) & 20 & 20 & - \\
\hline Power (kW) & 10 & 10 & - \\
\hline
\end{tabular}

Figure 4. Performances of the FEL based on LWFA.
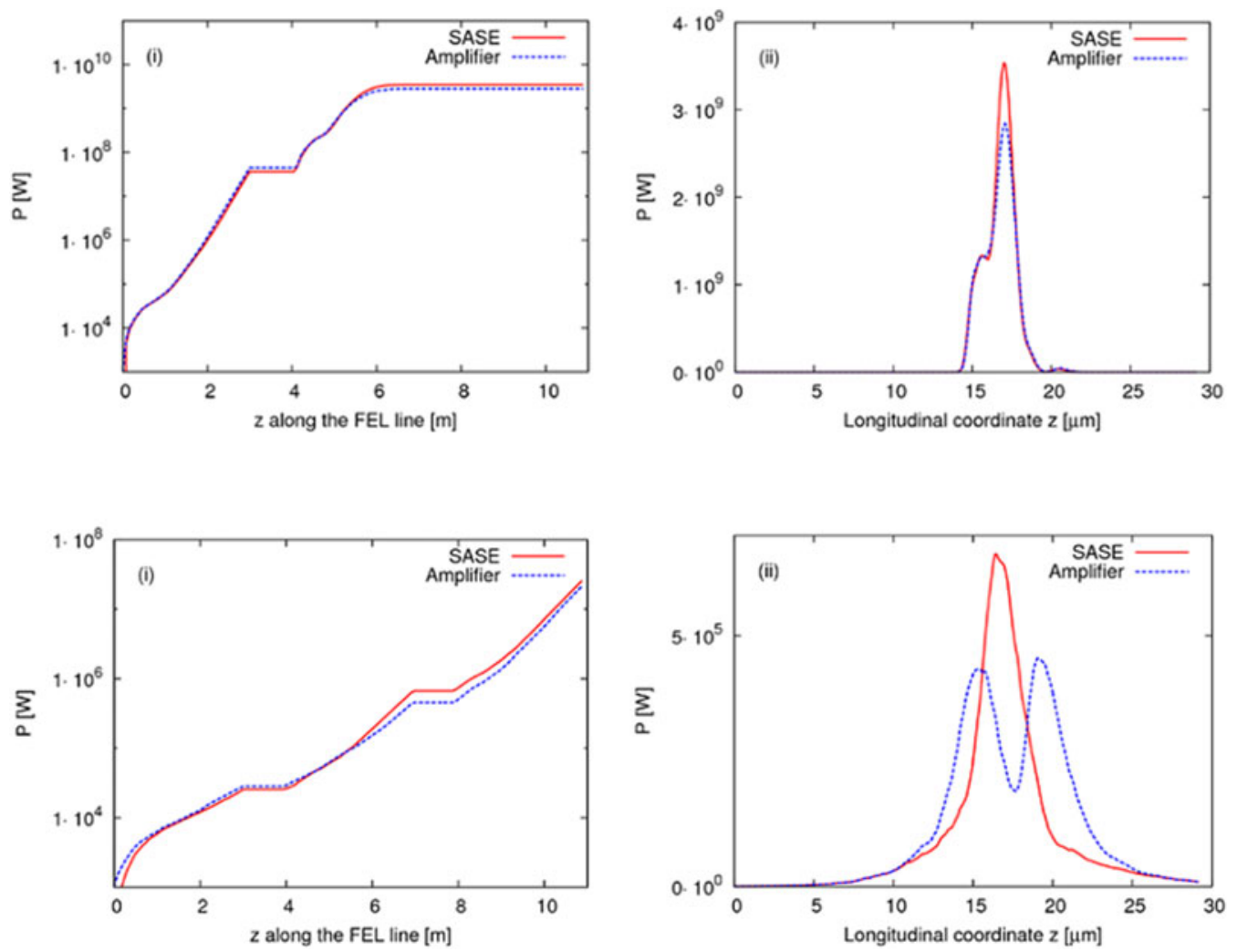

Figure 5. FEL performances @ $400 \mathrm{MeV}$ in SASE (red line) and Amplifier configuration (blue line). For amplifier configuration: seeding power is $1 \mathrm{~kW}$. (i) Output power along the radiator sections. (ii) Longitudinal power distribution at the exit of the second radiator. Electron bunch duration: a) 2 fs-rms and b) 20 fs-rms, peak current: a) $10 \mathrm{kA}$ and b) $1 \mathrm{kA}$, energy spread: $10^{-3}$. 


\section{Web of Conferences}

typically occurs in the transport line, because of the quadrupoles. Increasing the bunch duration from 2 to $20 \mathrm{fs}$, i.e. decreasing the peak current from 10 to $1 \mathrm{kA}$ leads to a decrease of the output power by more than two orders of magnitude at the second undulator exit. Saturation is no longer reached even within three sections. Increasing in addition the energy spread to $1 \%$ drives the radiation back to the level of spontaneous emission. Beam quality at the LWFA exit and its transport to the undulator are critical steps to be worked out on the path to LWFA based FELs.

\section{LUNEX5 EXPERIMENTAL STATIONS}

Pilot user experiments on isolated species and condensed matter will validate the LUNEX5 demonstrator radiation from a user point of view. So far, no user experiments have been carried out neither with HHG seeded FEL nor with Echo Enabled Harmonics. LUNEX5 has two end stations for time resolved pumpprobe studies of isolated species (TR-AMO) and for condensed matter imaging exploiting the coherence. The TR-AMO end station will consist in a high resolution velocity map imaging (VMI) spectrometer allowing for spectroscopy of cold atoms/molecules, clusters or nanoparticles, issued from a multipurpose source, combined with the full momenta characterisation of both electrons and ions using a COLTRIMS type of spectrometer based on time-of-flight and particle 2D position detection (covariance mapping will be used for data analysis). Lensless imaging technique also relies on very stable setup and accurate algorithms to extract real space images. In first experiments magnetization dynamics will be triggered through intense fs laser irradiation and then probed by resonant x-ray magnetic scattering.

\section{CONCLUSION}

LUNEX5 is aiming for ultra-short FEL pulses quest, production and use on a coupled CLA-LWFA flexible based test facility for complementary use and test of new ideas, such as the recently proposed scheme [22] with three electron photon interactions which could enable to reach the $1 \mathrm{~nm}$ region. It will pave the way towards the next generation of light source (4GLS+, 5GLS).

\section{References}

[1] Deacon D.A.G. et al., Phys. Rev. Lett. 38, 892-894 (1977)

[2] P. Emma et al., Nature Photonics 4, 641 (2010)

[3] T. Shintake, Proceed. FEL11 conference, Shanghai, Aug. (2011)

[4] R. Bonifacio, C. Pellegrini, L.M. Narducci, Optics Comm. 50, 373-378 (1984)

[5] Y. Din et al., Phys. Rev. Lett. 102, 254801 (2009)

[6] P. Emma, Proceed. IPAC 12, New Orleans, USA, (2012)

[7] L.H. Yu et al., Science 289, 932 (2000)

[8] Lambert G et al., Nature Physics 4, 296-30 (2008)

[9] T. Togashi et al., Optics Express 1, 317-324 (2011)

[10] G. Stupakov G et al., Phys. Rev. Lett. 102, 074801 (2009)

[11] D. Xiang et al., Phys. Rev. Lett. 105, 114801 (2010)

[12] Zhao Z.T. et al., Proceed. FEL10 Conference, Malmö, Sweden, 25-27 August, 15-19 (2010)

[13] O. Lundh et al., Nature Physics 7, 219-222 (2011)

[14] K. Nakajima, Nature Physics 4, 92 (2008)

[15] W. Leeman, E. Esarey E., Phys. Today 62, 3, 44 (2009)

[16] H.P. Schlenvoigt et al., Nature Physics 4, 130-133 (2008)

[17] M. Fuchs et al., Nature Physics 5, 826 (2009)

[18] W.M. Fawley et al., Proceed. FEL06 Conference, Berlin, Germany, 455-458 (2006)

[19] M.P. Anania et al., Proceed. IPAC10, Kyoto, Japan, 2263-2265 (2010)

[20] http://www.mpq.mpg.de/APS/gruener.php 


\section{UVX 2012}

[21] D. Xiang and G. Stupakov, New J. Phys. 13,093028 (2011)

[22] DESY- PITZ Collaboration, http://pitz.desy.de/collaboration

[23] TESLA Technology Collaboration, http://tesla-new.desy.de/

[24] C. Benabderrahmane et al., Proceed. FEL11 Conference, Shanghai, China, (2011)

[25] H. Maesaka et al., Proceed. DIPACO9 Conference, Basel, Switzerland, (2009) 